\title{
Vascular Renal Anatomy and the Ureteropelvic Junction: Preoperative Multidetector CT Scanning with Split-Bolus Injection as a Predictor of Laparoscopic Findings*
}

\author{
BENOIT SAUER, M.D., ${ }^{1}$ MURIEL FLOCQUET, M.D., ${ }^{1}$ TOUFIK BATCH, M.D., ${ }^{1}$ \\ ALAIN BLUM, M.D., Ph.D., ${ }^{1}$ and JACQUES HUBERT, M.D., Ph.D. ${ }^{2}$
}

\begin{abstract}
Purpose: To compare multidetector CT scan (MDCT) results with intraoperative findings in the detection of an inferior-pole pedicle crossing the ureteropelvic junction.

Patients and Methods: Over the 2-year study period, 35 patients receiving laparoscopic pyeloplasty underwent preoperative investigation with a novel MDCT protocol in order to detect crossing vessels. Postprocessing, including maximum intensity projection, volume-rendering technique, and multiplanar reconstruction, was used in addition to standard axial views.

Results: All the arteries found during laparoscopic surgery were detected by MDCT, but one radiologic false-positive was noted at the beginning of the series. Seven veins were not detected with MDCT. In the only case featuring an isolated inferior-pole vein, the aberrant vessel was identified by MDCT.

Conclusion: Multidetector CT scanning is a highly accurate way of providing all the information necessary preoperatively concerning renal parenchymal anomalies, urinary stones, and collecting system and vessel anatomy. It helps physicians make appropriate therapeutic decisions and gives surgeons information about what they can expect during laparoscopic procedures.
\end{abstract}

\section{INTRODUCTION}

$\mathbf{P}$ REOPERATIVE INFORMATION about a patient's renal anatomy is likely to reduce the risk of problems during laparoscopy for ureteropelvic junction (UPJ) obstruction. Detection of an inferior-pole vessel (which may cause failure or hemorrhagic complications during endopyelotomy) would be particularly helpful in ensuring appropriate medical and surgical intervention.

The purpose of this study was to determine whether the findings of a multidetector CT scan (MDCT) would facilitate surgical management of patients with symptomatic UPJ obstruction. Specifically, we assessed prospectively the ability of preoperative MDCT to detect an inferior-pole vessel crossing the UPJ.

\section{PATIENTS AND METHODS}

Between November 2001 and September 2003, 35 patients (mean age 40 years; range 18-77 years) scheduled for laparoscopic robot-assisted pyeloplasty were examined preoperatively for a crossing vessel using multiphasic MDCT (16 rows, Sensation $16^{\circledR}$, Siemens, Erlangen, Germany). Multidetector CT was conducted in three steps: a nonenhanced scan followed by administration of $60 \mathrm{~mL}$ of iodinated contrast medium (iodixanol [Vispaque ${ }^{\circledR}$ 320]; Amersham Health SA) and then immediate intravenous injection of a diuretic (furosemide $80 \mathrm{mg}$ ). Ten minutes later, CT acquisitions were obtained in the arterial phase (30 seconds after the start of a second injection of the same contrast medium) and the venous (nephrographic) phase (100 seconds after the second injection of contrast medium).

\footnotetext{
${ }^{1}$ Service d'imagerie Guilloz, Hôpital central, CHU Nancy, France.

${ }^{2}$ Service d'urologie, Hôpitaux de Brabois, CHU Nancy.

* Entry in the 2006 Endourological Society Clinical Essay Competition.
} 
The following CT parameters were of interest: $16 \times 1.5 \mathrm{~mm}$ in the unenhanced phase and $16 \times 0.75 \mathrm{~mm}$ in the arterial and nephrographic phases. Particular attention was paid to radiation dose. The ALARA (as low as reasonably achievable) protocol was adopted. ${ }^{1}$ For nonenhanced CT, $120 \mathrm{kV}$ with $\mathrm{mAs}$ equal to body weight, and for enhanced CT, $120 \mathrm{kV}$ with mAs equal to three times body weight, were used. Established values of CTDIvol were approximately $5 \mathrm{mGy}$ for the nonenhanced phase and $12 \mathrm{mGy}$ for the enhanced phases.

Axial images were reconstructed using a standard abdominal filter with an effective slice thickness of $1 \mathrm{~mm}$ and a slice interval of $0.7 \mathrm{~mm}$ in the arterial and nephrographic phases. Unenhanced acquisition involved 2-mm thick slices with a 1.5mm slice interval.

All imaging data were viewed and analyzed by consensus on an imaging workstation (Leonardo ${ }^{\circledR}$ [Siemens] or Voxar ${ }^{\circledR}[$ Barco, Kortrijk]) by two experienced radiologists. Multiplanar re-formations were obtained in coronal and sagittal views. Other postprocessing included maximum intensity projection (MIP), multiplanar volume rendering (MPVR), and volume-rendering technique (VRT). Radiologic analysis was conducted on axial images, and three-dimensional (3D) reconstructed images (VRT, MPVR). Perioperative laparoscopic videos were reviewed by a single urologist $(\mathrm{JH})$; these images were considered the gold standard and compared with the radiologic findings.

The time needed for the entire procedure was measured from the first scout view through reconstruction of the last image.

\section{RESULTS}

The MDCT protocol was quick (15 minutes) and generally well accepted by patients. No adverse reactions were observed after contrast or furosemide injection.

The primary objective was to evaluate the ability of MDCT to identify crossing vessels accurately. Among the 35 patients, MDCT highlighted 12 single polar arteries, 1 single vein, and 11 polar pedicles (artery and vein)(Figs. 1-4). The corresponding operative findings were 4,1 , and 18, respectively. All the arteries observed during the laparoscopic procedure had been detected on MDCT. One radiologic false-positive was noted. Seven veins associated with a polar artery were missed by MDCT, but the only case of an isolated inferior polar vein was detected. There were no cases in which a posterior inferior pedicle was closely related to the UPJ. The sensitivity and specificity of MDCT in depicting a polar artery thus were $100 \%$ and $92.3 \%$, respectively, whereas the sensitivity and specificity in depicting polar veins were $63 \%$ and $100 \%$, respectively.

\section{DISCUSSION}

\section{Physiopathology of UPJ obstruction}

Ureteropelvic junction obstruction may be attributed to intrinsic or extrinsic causes. A combination of muscular defects, excessive collagen deposits, and abnormal intercellular conduction has been implicated in the common form of intrinsic obstruction. Factors associated with extrinsic obstruction include fixed kinks or angulations, bands of tissue, high insertion of the ureter on the renal pelvis, and crossing vessels. Secondary UPJ obstruction may be related to infection, stones, surgery, ischemia, and iatrogenic injury. The functional significance of crossing vessels at the site of obstruction is controversial. Although some reports claim that crossing vessels have been implicated in UPJ obstruction, these vessels more commonly aggravate rather than cause the obstruction. ${ }^{2-8}$ Computed tomography scanning is the only technique that can provide all the necessary information in a single examination.

\section{Treatment of UPJ obstruction}

Treatment for UPJ obstruction differs from patient to patient depending on the cause. Open dismembered pyeloplasty is still considered the gold standard and is associated with the highest rate of success (as high as $95 \%$ ). ${ }^{2}$ A variety of minimally invasive therapeutic options have been developed, including an endoscopic antegrade or retrograde approach in which the UPJ obstruction is visually or radioscopically identified and incised (Acucise ${ }^{\circledR}$ Applied Medical, Laguna Hills, CA). However, these techniques have a $10 \%$ to $20 \%$ lower success rate than open surgery and are associated with a higher risk of retroperitoneal hemorrhage. Blood transfusion is required in $1 \%$ to $3 \%$ of patients when an inferior renal pedicle is not recognized. Highgrade hydronephrosis, poor renal function, high insertion of the ureter, and the presence of a crossing vessel are prognostic of endopyelotomy failure. ${ }^{2,7}$

A clear preoperative understanding of the patient's renal anatomy is of great value to urologists. Previous anatomic investigations have shown that vessels have a close relation to the UPJ in approximately $75 \%$ of patients with the syndrome. $^{3,4,8,9}$ This rate was confirmed here, with $63 \%$ of the present series exhibiting an inferior-pole pedicle.

Laparoscopic pyeloplasty differs from the open technique only in the endoscopic approach. Positive results have been achieved in expert hands, but the procedure is demanding and has a long learning curve. By providing a 3D view, unprecedented control of the endocorporeal instruments, and optimum working condition and good ergonomics for the surgeon, robots such as the da Vinci ${ }^{\circledR}$ Surgical System may allow urologists with limited laparoscopic experience to master the endocorporeal management of UPJ obstruction rapidly. This is likely to lead to minimally invasive urologic techniques becoming increasingly popular. ${ }^{2}$

In our institution, patients with UPJ obstruction typically are given the opportunity to choose between laparoscopic robotic pyeloplasty and endopyelotomy. They are informed that the open and laparoscopic approaches both have high success rates but that the former involves slightly greater morbidity and potential complications because of the incisions. Patients also are informed of the risk of hemorrhage associated with endopyelotomy. However, if the patient is initially inclined toward endoscopic treatment and has not undergone contrast-enhanced abdominal CT or MRI, we order a UPJ-protocol CT scan. In our experience, crossing vessels contraindicate the endoscopic approach. Thus, fewer than $40 \%$ of patients could benefit from endoscopic treatment. Moreover, most of the remaining patients have high-grade hydronephrosis, high insertion of the ureter, or renal stones, all of which are prognostic of endopyelotomy failure. ${ }^{7}$ Because of these factors, most of the patients in our in- 


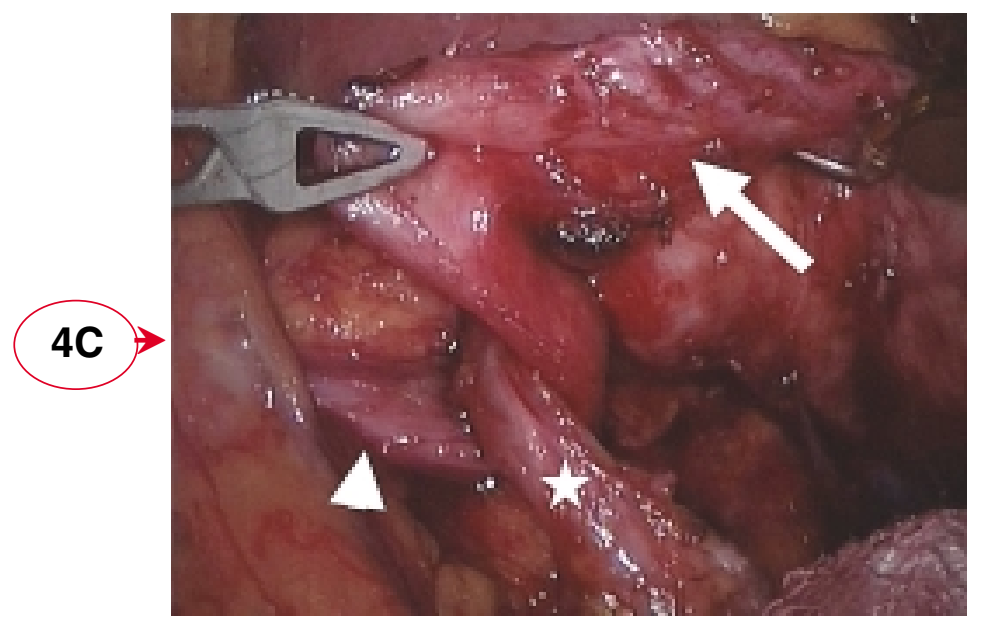

FIG. 1. Preoperative view of UPJ. White arrow indicates renal pelvis, arrowhead shows ureter, and star marks crossing vessel.

stitution undergo robotic laparoscopic surgery. In our experience with nearly 100 cases, the success rate is high, even in complex situations such horseshoe kidneys. ${ }^{10}$

\section{Accuracy of MDCT}

The high speed of MDCT enables anatomic regions to be scanned in a single breathhold with minimal ventilatory or other motion artifacts. High-quality MPVR and other postprocessing depictions such as MIP and VRT can be generated, and all images are viewed on a workstation. These advantages have enhanced the imaging of small vessels. ${ }^{3,4,8,11,12}$

In our study, all the arteries observed during the laparoscopic approach were detected by MDCT. Classically, digital subtraction angiography (DSA) with selective injections is considered to be the standard of reference in depicting polar arteries. Rouviere and colleagues ${ }^{9}$ compared the accuracy of DSA and renal CT for detecting polar arteries in 41 patients. The CT

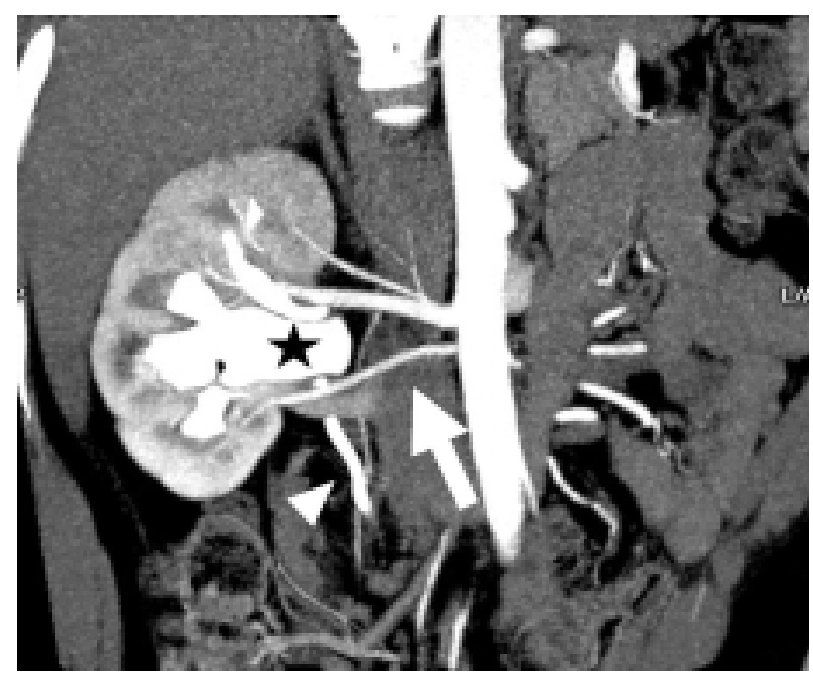

FIG. 2. Oblique coronal view of UPJ obstruction. Note crossing vessel (arrow) between renal pelvis (star) and ureter (arrowhead).

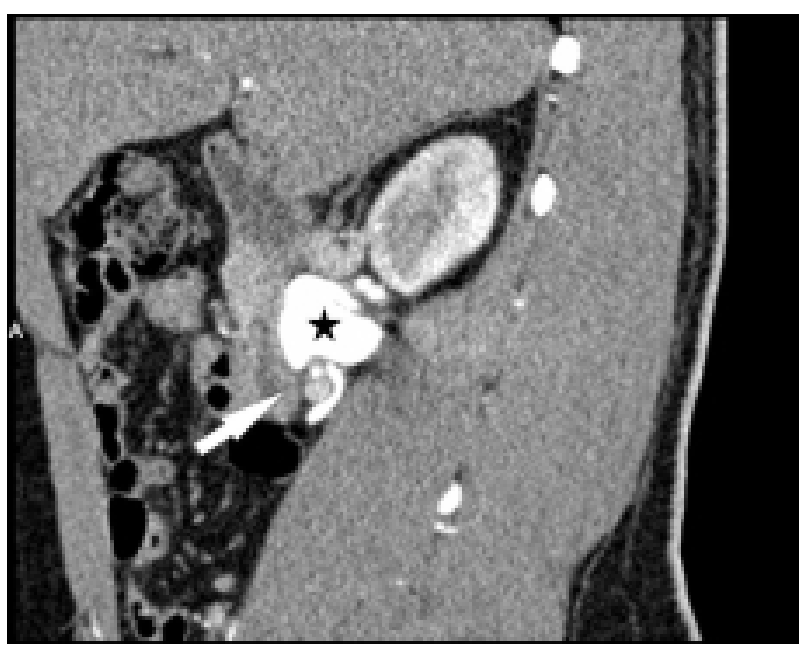

FIG. 3. Sagittal MPR view of right renal hilum showing kinking of UPJ (star) over inferior-pole pedicle (arrow).

angiography study depicted $121(96 \%)$ of the 126 renal arteries prospectively; and retrospectively, 124 renal arteries (98\%) were visible on the CT images. ${ }^{9}$ Moreover, MDCT technology has improved since 1999, and even better results are to be expected with 16-row or 64-row scanners.

Multidetector CT is less expensive and less invasive than DSA. Multidetector CT angiography can not only highlight the position of the UPJ and the proximal ureter in most patients with UPJ obstruction but also depicts the vascular anatomy at the UPJ and the renal-pelvic size and helps to rule out renal calculi, parenchymal lesions, and congenital anomalies such as duplicated ureter. The relations of blood vessels to nonvascular structures, such as the renal pelvis and ureter, can be shown more accurately with MDCT angiography than with conven-

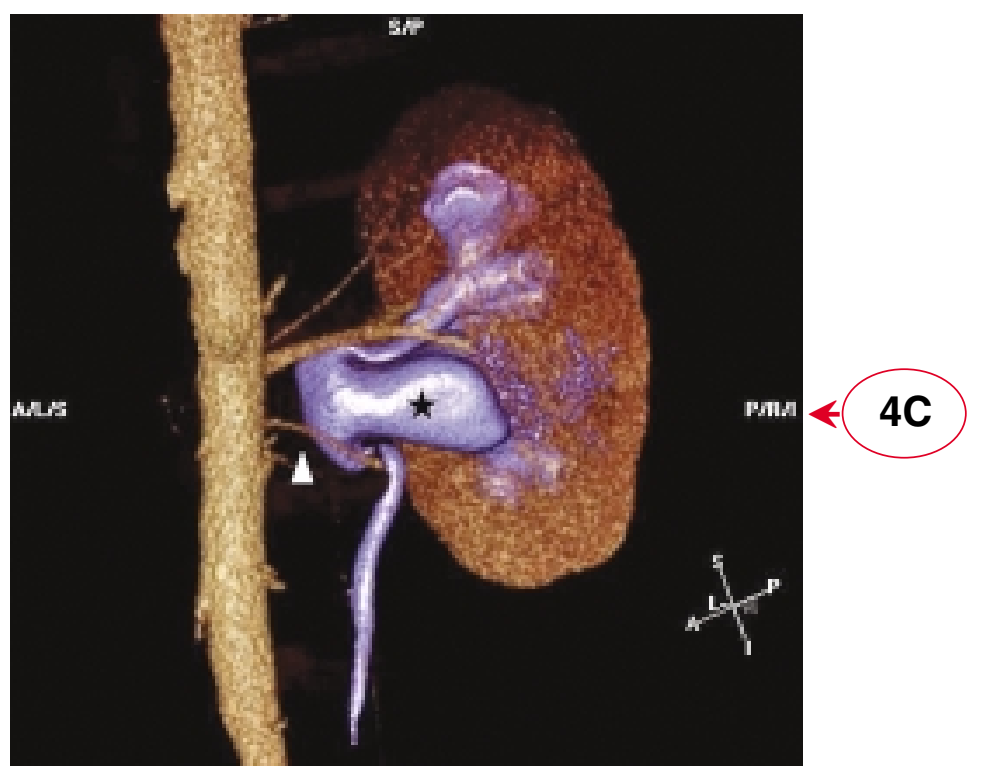

FIG. 4. Posterolateral VRT (on Voxar ${ }^{\circledR}$ ) view of UPJ. Note kinking of urinary tract (star) over inferior-pole pedicle (arrowhead). 
tional angiography. ${ }^{3,4,9}$ However, the literature provides few data on correlations between MDCT and surgical findings; our study showed an excellent correlation.

\section{Split-bolus injection of iodinated contrast medium}

Multidetector CT urography allows "all-in-one" evaluation of potential calculi and renal parenchymal masses and may depict urothelial lesions. It is the most accurate way with which to determine the renal-parenchymal thickness, the size and the anatomy of the UPJ, and, particularly, the insertion of the ureter. A variety of CT techniques and protocols are currently used, and no specific method has gained widespread acceptance. ${ }^{13}$

Our MDCT protocol is rapid and well accepted by patients. A split-bolus injection was used to permit simultaneous CT angiography and urography, thereby enabling informative 3D reconstruction to be carried out. Similarly, Chow and coworkers $^{14}$ described using split-bolus IV contrast injection to obtain simultaneous nephrographic- and excretory-phase images of the urinary tract. With this split-bolus injection, late-phase (classical excretory-phase) investigation is unnecessary, reducing the radiation exposure.

Simultaneous angiographic- and excretory-phase examination does not improve the depiction of polar vessels on multiplanar reconstruction. However, our protocol with previous opacification of the collecting urinary system highlights the relations between the crossing vessel and the UPJ on MIP and VRT images: a very helpful feature for the surgeon. Although this protocol requires a higher total volume of contrast material, no renal failure was observed in our 35 patients.

An increase in the size of the renal pelvis as seen on urography after injection of diuretics is diagnostic of UPJ. We therefore recommend administering the injection and waiting about 10 minutes between the phases. Thus, this protocol provides morphologic and dynamic information. It can also depict intermittent UPJ obstruction but probably is not required in all cases and might best be used selectively, depending on the degree of pelvic distention on the initial nonenhanced scans. Diuretic injection in patients with UPJ obstruction occasionally leads to renal colic pain; however, this responds rapidly to antispasmodic and anti-inflammatory drugs. ${ }^{3,4}$

There is, as yet, no widely accepted dedicated CT urography protocol for optimal opacification and distention of the collecting system. ${ }^{13}$ The injection of a diuretic leads to rapid and uniform distribution of contrast material through the urine and is more efficient than other procedures such as saline serum injection. ${ }^{15} \mathrm{We}$ advocate the use of a diuretic in order to decrease streaking artifacts that lead to a partial loss of caliceal and adjacent vessel details. Opacification of the collecting system appears more uniform and free of dense contrast material stasis within the papillae and calices when diuretic is injected. ${ }^{15}$

\section{MDCT compared with other techniques}

Inferior-pole pedicles can be detected with external ultrasonography, but the image quality depends on the expertise of the operator and the anatomy of the patient. Endoluminal ultrasonography can define the adjacent vasculature only close to the surface around the UPJ because of the high frequency of the probe. However, this procedure is carried out under general anesthesia and involves fluoroscopy-guided retrograde ureteral catheterization. ${ }^{16}$ As it is an invasive diagnostic examination performed at the time of the surgical repair of the UPJ, the information it provides cannot be discussed beforehand when decisions are being made about the appropriate surgical technique. It is not widely used at present. ${ }^{2,7}$

Magnetic resonance angiography can be used to depict the renal vasculature. Using DSA as the reference standard, Bakker and collaborators ${ }^{17}$ identified 21 of 22 accessory renal arteries and Korst et al ${ }^{18}$ identified 13 of 17 . There are a few reports of detection of a crossing vessel around the UPJ using MRI. ${ }^{19}$ However, small $(<2-\mathrm{mm})$ accessory arteries and branches may not be seen with the limited spatial resolution of current MR sequences. Magnetic resonance imaging may have value in determining single-kidney function and evaluating urinary excretion. ${ }^{20}$

\section{Accuracy of detection of veins}

When isolated, a polar vein is of clinical importance and must be highlighted. When adjacent to an inferior-pole artery, its presence does not modify the surgical procedure, and therefore its detection is less crucial. ${ }^{2,7}$ In our study, we missed seven veins in the crossing pedicle, but in the only case with an isolated inferior polar vein, the aberrant vessel was identified on MDCT. Some authors have argued that arteries can theoretically be distinguished from veins using a dual-phase technique and small-interval reconstruction. ${ }^{3,4}$ However, small veins remain difficult to identify, as in the present study. To the best of our knowledge, the only reports concerning detection of variation in renal veins involved potential living kidney donors, and the analysis was limited specifically to circumaortic veins, multiple hilar veins, or anastomoses with lumbar veins. ${ }^{21}$ Polar veins in crossing pedicles were not evaluated. In our study, $51 \%$ of patients had crossing veins, a higher percentage than was expected considering previous results. To our knowledge, there are no reports in the literature in which MDCT was used to detect a vein in a crossing pedicle. Nevertheless, crossing arteries and veins have been differentiated..$^{3,4,8}$ Moreover, MDCT is more accurate than DSA when highlighting a renal polar vein. ${ }^{9}$ The only isolated inferior polar vein in our series was documented and described as a vein and not an artery. The presence or absence of a vein adjacent to an inferior-pole artery in a pedicle did not modify the surgical procedure.

\section{CONCLUSION}

Multidetector CT scanning is a highly accurate means of detecting inferior-pole arteries preoperatively but is of less value in the depiction of polar veins, particularly when associated with an artery. It provides significant information to indicate surgery and helps surgeons anticipate difficulties. Our protocol combines CT angiography and urography with split-bolus contrast medium injection and IV diuretic. It provides a highly uniform opacification and good distention of pelvis and calices, thereby facilitating postprocessing. Radiation exposure also is lower. In a single examination, MDCT provides all the information necessary to plan the surgical procedure, covering renal parenchymal anomalies, urinary stones, and collecting-system and vessel anatomy. 


\section{REFERENCES}

1. European Commission. European Guidelines on Quality Criteria for Computed Tomography. Luxemburg: The Commission, 1999.

2. Hubert J. Robotic pyeloplasty. Curr Urol Rep 2003;4:124.

3. Lawler LP, Jarret TW, Corl FM, et al. Adult ureteropelvic junction obstruction: Insights with three-dimensional multi-detector row CT. Radiographics 2005;25:121.

4. Mitsumori A, Yasui K, Akaki S, et al. Evaluation of crossing vessels in patients with ureteropelvic junction obstruction by means of helical CT. Radiographics 2000;20:1383.

5. Munver R, Sosa RE, del Pizzo JJ. Laparoscopic pyeloplasty: History, evolution, and future. J Endourol 2004;18:748.

6. Sampaio FJ, Favorito LA. Ureteropelvic junction stenosis: Vascular anatomical background for endopyelotomy. J Urol 1993;150: 1787.

7. Van Cangh PJ, Nesa S, Galeon M, et al. Vessels around the ureteropelvic junction: significance and imaging by conventional radiology. J Endourol 1996;10:111.

8. Quillin SP, Brink JA, Heiken JP, et al. Helical (spiral) CT angiography for identification of crossing vessels at the ureteropelvic junction. AJR Am J Roentgenol 1996;166:1125.

9. Rouviere O, Lyonnet D, Berger P, et al. Ureteropelvic junction obstruction: Use of helical CT for preoperative assessment-Comparison with intraarterial angiography. Radiology 1999;213:668.

10. Chammas M, Jr., Feuillu B, Coissard A, et al. Laparoscopic robotic-assisted management of pelvi-ureteric junction obstruction in patients with horseshoe kidneys: Technique and 1-year follow-up. BJU Int 2006;97:579.

11. Blum A, Ludig T, Coll D, et al. [Preoperative evaluation of renal carcinoma using multi-detector CT]. J Radiol 2002;83:431.

12. Gimel P, Hubert J, Iochum S, et al. [Contribution of the latest generation CT in preoperative assessment of kidney cancer]. Prog Urol 2002; $12: 1310$.

13. Korobkin M. CT urography. Eur Radiol 2005;15(suppl 4):D82.

14. Chow LC, Sommer FG. Multidetector CT urography with abdominal compression and three-dimensional reconstruction. AJR Am J Roentgenol 2001;177:849.

15. Kemper J, Regier M, Begemann PG, et al. Multislice computed tomography-urography: Intraindividual comparison of different preparation techniques for optimized depiction of the upper urinary tract in an animal model. Invest Radiol 2005;40:126.

16. Siegel CL, McDougall EM, Middleton WD, et al. Preoperative assessment of ureteropelvic junction obstruction with endoluminal sonography and helical CT. AJR Am J Roentgenol 1997;168:623.
17. Bakker J, Beek FJ, Beutler JJ, et al. Renal artery stenosis and accessory renal arteries: accuracy of detection and visualization with gadolinium-enhanced breath-hold MR angiography. Radiology 1998;207:497.

18. Korst MB, Joosten FB, Postma CT, et al. Accuracy of normal-dose contrast-enhanced MR angiography in assessing renal artery stenosis and accessory renal arteries. AJR Am J Roentgenol 2000;174: 629.

19. McDaniel BB, Jones RA, Scherz H, et al: Dynamic contrast-enhanced MR urography in the evaluation of pediatric hydronephrosis 2: Anatomic and functional assessment of ureteropelvic junction obstruction. AJR Am J Roentgenol 2005;185:1608.

20. Rohrschneider WK, Haufe S, Wiesel M, et al. Functional and morphologic evaluation of congenital urinary tract dilatation by using combined static-dynamic MR urography: findings in kidneys with a single collecting system. Radiology 2002;224:683.

21. Kawamoto S, Lawler LP, Fishman EK. Evaluation of the renal venous system on late arterial and venous phase images with MDCT angiography in potential living laparoscopic renal donors. AJR Am J Roentgenol 2005;184:539.

Address reprint requests to: Benoit Sauer, M.D.

Service d'imagerie Guilloz

Hôpital central

Avenue de Lattre de Tassigny 54000 Nancy, France

E-mail: benoit.sauer@neuf.fr

\section{ABBREVIATIONS USED}

$3 \mathrm{D}=$ three-dimensional; DSA $=$ digital subtraction angiography; IV = intravenous; $\mathrm{MDCT}=$ multidetector computed tomography; $\mathrm{MIP}=$ maximum intensity projection; $\mathrm{MPVR}=$ multiplanar volume rendering; $\mathrm{MRI}=$ magnetic resonance imaging; UPJ = ureteropelvic junction; VRT = volume-rendering technique. 
